Article

\title{
The Secrets of Beautiful Hair: Why is it Flexible and Elastic?
}

\author{
Mikako Ezure *(D), Noriyuki Tanji, Yukari Nishita, Takashi Mizooku, Shinobu Nagase and \\ Takahiro Osumi
}

R\&D-Hair Care Products Research, Kao Corporation, 2-1-3, Bunka, Sumida-ku, Tokyo 131-8501, Japan

* Correspondence: ezure.mikako@kao.com; Tel.: +81-3-5630-9714

Received: 4 June 2019; Accepted: 23 June 2019; Published: 6 July 2019

\begin{abstract}
Beautiful hair, so called "SHINAYAKA" hair in Japanese, has a good appearance not only when stationary but also when in motion, and it is a highly desirable hair condition for Japanese consumers. We investigated such SHINAYAKA hair, which was selected by sensory evaluation, for the relationship between physical properties, such as flexibility and elasticity, and hair structure. It has already been reported that human hair cortical cells have two types, similar to wool: the ortho-like cortex and the para-like cortex. Microscopic observation revealed that the ortho-like cortex is distributed in the outer layer of the hair (near the hair surface) and the para-like cortex exists in the inner layer (near the center of the fiber). This cell distribution, a concentric double-layered structure, was deemed to be a characteristic of SHINAYAKA hair. Furthermore, analysis of physical properties showed the difference between the elasticity of the outer layer and inner layer, and that this difference was bigger in SHINAYAKA hair compared to other hair. This phenomenon was observed not only in Japanese hair, but also in Caucasian hair. In addition, we have developed a new technology for creating "SHINAYAKA" hair by treatment with succinic acid. Inflexible and inelastic hair can be changed by this treatment, and its flexibility and elasticity improve by selective reduction of stiffness of the outer layer.
\end{abstract}

Keywords: hair; flexible; elastic; dynamic; motion; ortho-like; para-like; cortex; bending; mechanical; succinic acid; concentric double-layered

\section{Introduction}

Beautiful hair has been desired since ancient times. For this purpose, for several decades we have investigated beautiful hair in depth, developing hair care technologies and launching various unique products for consumers in the world. As important elements for beautiful hair, hair shine, surface friction and hair shape are generally known. Based on our macroscopic and microscopic observations of the hair fibers and their structures, relationships between hair appearance and its structure were clarified. Well-ordered alignment of the hair fibers enhances the reflections by sharpening shine, and a smooth surface and pore-less inside structure contributes to a good appearance. Our products apply technologies developed on such knowledge. Some organic acids (e.g., malic acid, lactic acid, succinic acid) are able to repair the surface of the hair fiber, create a pore-less internal hair structure or alter the physical properties of the hair fiber [1-8].

In this study, we have focused on the motion of hair strands, especially beautiful movements, as if polar auroras were fluttering or green grasses were waving on a prairie in the wind. This motion of hair fibers is flexible and elastic like a fluid, but these fibers smoothly return to their original shape. Such flexible and elastic behaviors are traditionally preferred in Japan and described as "SHINAYAKA" in Japanese. In this report, thus flexible and elastic property is described as "flexible/elastic", while the opposite inflexible and inelastic property is described as "inflexible/inelastic". 
Several evaluation methods for hair motion have been reported [9-11]. These reports suggest that hair motion arises from damage and can be improved after treatment with a conditioner. They have not mentioned, however, the difference in the motion among individual hairs. The motion of a hair strand is a complex phenomenon, with the main contribution from hair-hair interactions, hair shape and physical properties of hair fibers. In the cases of virgin straight hair, the individual differences of the hair-hair interaction and hair shape are probably not so large, but a difference in the hair motion is, however, still observed among individuals.

We considered bamboo and a composite (multi-layer) bow as the models for a flexible and elastic fiber, because these are composite materials that have multipart mechanical properties such as flexible and elastic behavior. Bamboo is composed of hard elastic fibers (vascular bundles) and soft material (parenchyma cell) to absorb compressive deformation. A composite bow is composed of softer material in the convex side and harder material in the concave side. The bamboo can be deformed resiliently and flexibly without structural collapse [12]. The bow is easy to bend and returns to its original shape [13]. These composite materials can realize complex mechanical properties such as flexible and elastic behavior; however, it is difficult to realize such complex properties with a homogeneous material.

Hair fibers are also composite materials; they are composed of a scaly cuticle, a porous medulla and a fibrous cortex. The main component is the cortex, which makes up $85 \%$ of the hair fiber, and is composed of fibrous intermediate filaments (IFs) and keratin-associated proteins (KAPs). This means that the cortex is also a type of composite material. It is known that there are two types of cortices in wool. The ortho-cortex shows relatively small and dispersed macrofibrils in the fiber cross-section and a spiral alignment of IFs. On the other hand, the para-cortex shows relatively large and fused macrofibrils with a parallel alignment of the IFs. Similar structures were found in human hair [14-17]. These hair fiber physical properties are not simple.

In this study, we have investigated the relationship between the flexible/elastic behaviors of the hair fiber, the hair composite structure and its physical properties. Previously, we have partially reported this result [18], but here, in this report, we describe in detail the physical property changes due to hair damage and a new treatment. We have developed a useful treatment method using succinic acid, for both virgin and damaged hair, and demonstrate the effects of succinic acid on not only Japanese but also Caucasian hair.

\section{Materials and Methods}

\subsection{Hair Samples}

\subsubsection{Selection of Hair Samples}

Chemically untreated hair was obtained from 50 Japanese female volunteers. Ten typical hair samples that had variation in the flexible and elastic features were selected. These hair samples were classified into flexible/elastic and inflexible/inelastic hair, according to the following sensory tests. The sensory tests were performed by five special sensory evaluators in the laboratory. The viewpoints to evaluate were the softness feeling and the bounce when the hair was swinging. The softness and bounce correspond to the flexible and elastic behaviors, respectively. Each evaluator selected the softest and the bounciest sample, and the stiffest and the least bouncy sample out of ten samples. The former was regarded as 'score 1', and the latter was regarded as 'score 10'. Based on this standard, they evaluated these ten samples on this 1-10 scale. Finally, the average score of each sample was taken from five evaluators' scores (Table 1). 
Table 1. Results of the sensory evaluation.

\begin{tabular}{ccc}
\hline Sample No. & Sensory Score (SS) & Evaluation \\
\hline 1 & 1.6 & flexible/elastic \\
2 & 1.8 & flexible/elastic \\
3 & 3.1 & flexible/elastic \\
4 & 4.2 & somewhat flexible/elastic \\
5 & 4.3 & somewhat flexible/elastic \\
6 & 4.5 & somewhat flexible/elastic \\
7 & 7.1 & inflexible/inelastic \\
8 & 8.5 & inflexible/inelastic \\
9 & 9.0 & inflexible/inelastic \\
10 & 9.3 & inflexible/inelastic \\
\hline
\end{tabular}

\subsubsection{Chemical Treated Hair}

For colored hair, a section $25 \mathrm{~cm}$ from the root end of a Japanese female's hair that was treated once with hair color one year ago was used for experiments. As an example of heavily damaged hair, parts of the root and tip (30 cm from the root end) of a Japanese female's hair which was treated with hair color and permed once a month were used for the experiments.

\subsubsection{Caucasian Hair}

Eight Caucasian straight hair samples (chemically untreated, gender unknown) were purchased from Kerling International Haarrfabrik GmbH (Backnang, Baden-Württemberg, Germany), and the most flexible/elastic Caucasian hair was selected by the sensory tests as described in Section 2.1.1.

\subsection{Preparation of the Hair Sample}

\subsubsection{Preparation of the Model Damaged Hair}

A bleaching agent containing $3.33 \% \mathrm{H}_{2} \mathrm{O}_{2}$ and $0.68 \% \mathrm{NH}_{3}, \mathrm{pH}=10$ was used. Commercial shampoo and conditioner were used in this treatment. The bleaching agent was applied to the hair sample at a ratio of 1:1 for $30 \mathrm{~min}$ at room temperature, and then rinsed with water. The hair sample was washed with shampoo, treated with conditioner and dried using a hair dryer. Model damaged hair was obtained by treating undamaged hair with bleach one time followed by washing twelve times as describe above. This treatment was repeated four times, totaling in 4 bleaches and 48 washes.

\subsubsection{Preparation of the Surface-Removed Hair}

Surface-removed hair (Figure 1) was prepared by grinding with alumina beads ( $2-2.5 \mathrm{~mm}$ in diameter). The thickness of the removed surface layer was determined as approximately $5 \mu \mathrm{m}$ by microscopic observation. This thickness corresponds to the cuticle layer (approximately $2 \mu \mathrm{m}, 5-6$ sheets) plus the cortical cell layer (approximately $3 \mu \mathrm{m}$ ) near the hair surface, in the cases of the hair samples used in this study. The tip of the hair fiber was attached to a drum, and the drum was rotated in alumina beads. A strand of hair $10 \mathrm{~cm}$ in length was fixed at a position that was $7 \mathrm{~cm}$ from the rotation axis so that the tip of the hair is at the front in the rotation direction. It was stirred for 8-12 $\mathrm{h}$ at a rotational speed of $40 \mathrm{rpm}$. The hair samples were periodically removed and the diameter scanned with a light microscope. The surface-removed hair prepared in this way was used to measure the bending elastic modulus of the hair's inner layer [19]. 

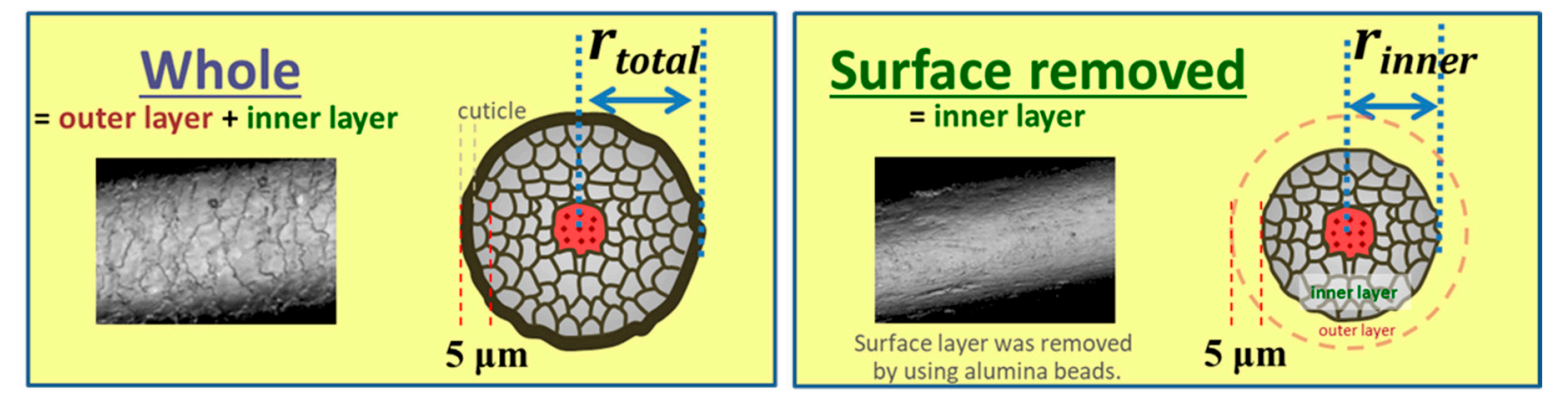

$$
r_{\text {total }}=r_{\text {inner }}+5(\mu \mathrm{m})
$$

Figure 1. Surface layer was removed by using alumina beads. $r_{\text {total }}=$ radius of whole hair, $r_{\text {inner }}=$ radius of surface removed hair.

\subsubsection{Treatment with Succinic Acid}

A hair sample was treated with $2.0 \%$ succinic acid (SA) solution, $\mathrm{pH}=3.7$, for $30 \mathrm{~min}$ at $40{ }^{\circ} \mathrm{C}$.

\subsubsection{The Three-Step Treatment System}

In order to provide realistic usage during in-bath conditions, a three-step treatment system was developed. The first step was just shampooing with a composition containing $11.5 \%$ ammonium polyoxyethylene(1.0) alkyl(C10-16) ether sulfates, $0.35 \%$ hydroxyethyl cellulose hydroxypropyl trimethylammonium chloride ether and $1.2 \%$ succinic acid, $\mathrm{pH}=3.9$. The second step was the application of the first conditioner containing $20 \%$ dipropylene glycol, $0.6 \%$ sodium 2-naphthalene sulfonate, $1.6 \%$ hydroxyethyl cellulose and $1.5 \%$ succinic acid, $\mathrm{pH}=6.0$. The third step was the application of the second conditioner containing $2.6 \%$ stearoxypropyl dimethylamine, $7.0 \%$ stearyl alcohol, $3.0 \%$ dimethicone and $0.5 \%$ amodimethicone, $\mathrm{pH}=3.4$, without rinsing off the first conditioner. This combination was left on the hair for $5 \mathrm{~min}$ and then finally all materials were rinsed off.

\subsection{Microscopic Observation Of Hair Cross-Sections}

\subsubsection{Transmission Electron Microscopy (TEM)}

Hair cross-sections were prepared using a microtome and observed using a transmission electron microscope (TEM) (H-7100; Hitachi High-Technologies Corporation, Minato, Tokyo, Japan) to determine the structural difference in cortical cell distribution between the flexible/elastic and inflexible/inelastic hairs. Hair samples were rinsed in ethanol to clean their surface and then dried before being put in embedding molds. Hair samples embedded in Spurr's resin (Spurr Low-Viscosity Embedding Media; Polysciences Inc., Warrington, Pennsylvania, USA) were sliced with a microtome (Leichert Ultracut N; Leica Microsystems, Wetzalar, Germany). The thickness of the hair section was $200 \mathrm{~nm}$. Then, the section was stained with $0.2 \%$ uranyl acetate solution, left for $10 \mathrm{~min}$ and rinsed with deionized water. Next, it was stained with lead citrate solution (Sigma-Aldrich, St. Louis, Missouri, USA) containing lead nitrate (II) and lead acetate (II) trihydrate, left for $10 \mathrm{~min}$, rinsed with deionized water and dried naturally. The TEM observation was performed at an acceleration voltage of $85 \mathrm{kV}$.

\subsubsection{Fluorescent Light Microscope (FLM)}

The cortical cell distribution was also confirmed with a differential staining method. Hair cross-sections of $5 \mu \mathrm{m}$ thicknesses were prepared and sequentially stained with sodium fluorescein and sulforhodamine 101 [14]. The hair samples were placed on slide glass and a few drops of $0.002 \%$ fluorescein sodium phosphate buffer solution was applied. After being left for $18 \mathrm{~h}$ under conditions of darkness and high humidity so as not to dry out, the samples were rinsed with deionized water 6 times and dried naturally for $20 \mathrm{~min}$. Then, drops of $0.005 \%$ sulforhodamine 101 solution phosphate buffer were applied and left for $1.5 \mathrm{~h}$ under the same conditions above, rinsed with deionized water 6 times, and dried. Para- and 
ortho-like cortical cells in the hair sections were differentially stained with the green fluorescein and red sulforhodamine, respectively. The stained hair samples were observed using a fluorescent light microscope (FLM) (Carl Zeiss AG, MPM 800, fluorescent filter: BP450-490/FT 510/LP520; Carl Zeiss AG, Oberkochen, Baden-Württemberg, Germany).

\subsection{Measurement of Bending Elasticity in the Outer and Inner Layers}

Hair fibers that were equilibrated at $20{ }^{\circ} \mathrm{C} / 65 \%$ relative humidity $(\mathrm{RH})$ for $24 \mathrm{~h}$ were used. The bending elasticity of each hair fiber was measured under the same conditions and 20 hair fibers were used for each measurement. The bending elasticity in both the minor and major axes of the hair samples were measured using a fiber bending measurement system (FBS900/FDAS765; Dia-Stron Limited, Andover, Hampshire, UK).

Bending elasticity (bending stress normalized with curvature and moment) was obtained as follows according to the general material dynamics relationship formula [20].

The following formula describes the relationship between bending stress (M), bending elasticity (E), the second moment of cross-section (I) and the radius of curvature ( $\rho$ ) (Equation (1)).

$$
\mathrm{M}=\mathrm{E} \times \mathrm{I} / \rho,
$$

The hair cross section is regarded as an ellipse; the radius on the major axis side is $R$, and the radius on the minor axis side is $r$. Assuming that the hair is bent to the minor axis side, the second moment of cross section of the elliptic cylinder with one end fixed is $I=\pi \operatorname{Rr}^{3} / 4$. When the hair is deformed as shown in Figure 2 as an end-loaded cantilever, the bending elasticity (E) is given by Equation (2) [21].

$$
\mathrm{E}=4 \mathrm{ML}^{3} / 3 \pi \operatorname{Rr}^{3} \mathrm{~d},
$$

where $\mathrm{L}$ is sample length and $\mathrm{d}$ is deflection due to the bending stress.

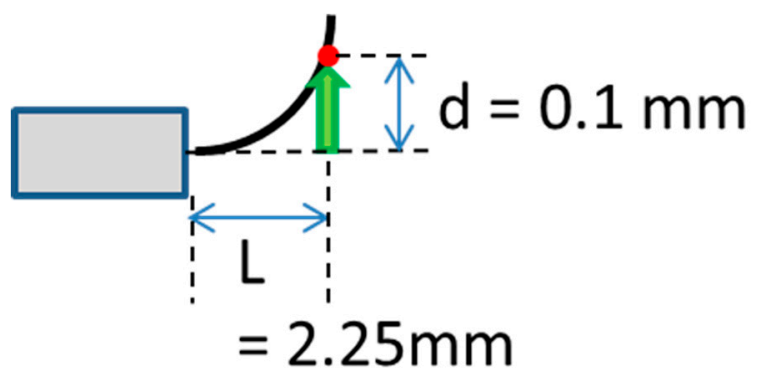

Figure 2. Schematic diagram showing measurement image of bending elastic modulus of hair. $\mathrm{L}=$ sample length; $\mathrm{d}=$ deflection due to bending stress.

First, when a load of $2 \mathrm{mg}$ was detected, d was set to $0 \mathrm{~mm}$, and deformation was performed from $\mathrm{d}=0$ to $0.1 \mathrm{~mm}$ at a speed of $0.01 \mathrm{~mm} / \mathrm{s}$.

In order to determine the mechanical properties in the outer and inner layers, a double-layered cylinder model was adopted. The bending elasticity of the whole hair fiber $\left(E_{\text {whole }}\right)$ can be described by Equation (3) (Figure 3) [22]:

$$
E_{\text {whole }}=E_{\text {outer }}\left(1-\frac{R_{\text {inner }} r_{\text {inner }}^{3}}{R_{\text {total }} r_{\text {total }}^{3}}\right)+E_{\text {inner }} \frac{R_{\text {inner }} r_{\text {inner }}^{3}}{R_{\text {total }} r_{\text {total }}^{3}}
$$

where $E_{\text {outer }}$ and $E_{\text {inner }}$ are the bending elasticities of the outer and inner layers (surface-removed hair), $R_{\text {total }}$ and $R_{\text {inner }}$ are the hair radii in the major axis of the intact whole hair and surface-removed hair, $r_{\text {total }}$ and $r_{\text {inner }}$ are the hair radii in the minor axis of the intact whole hair and surface-removed hair, respectively, and bending direction is assumed to be in the direction of the minor axis. $E_{\text {whole }}, E_{\text {inner, }}$ 
$R_{\text {total }}, R_{\text {inner }}, r_{\text {total }}$ and $r_{\text {inner }}$ are measurable, therefore $E_{\text {outer }}$ can be calculated according to the above Equation (3). Twenty fibers were randomly selected from each individual and the bending properties of whole hair and surface-removed hair were measured. Then, the average values of the bending elasticities in the outer and inner layers of flexible/elastic and inflexible/inelastic hairs were obtained.

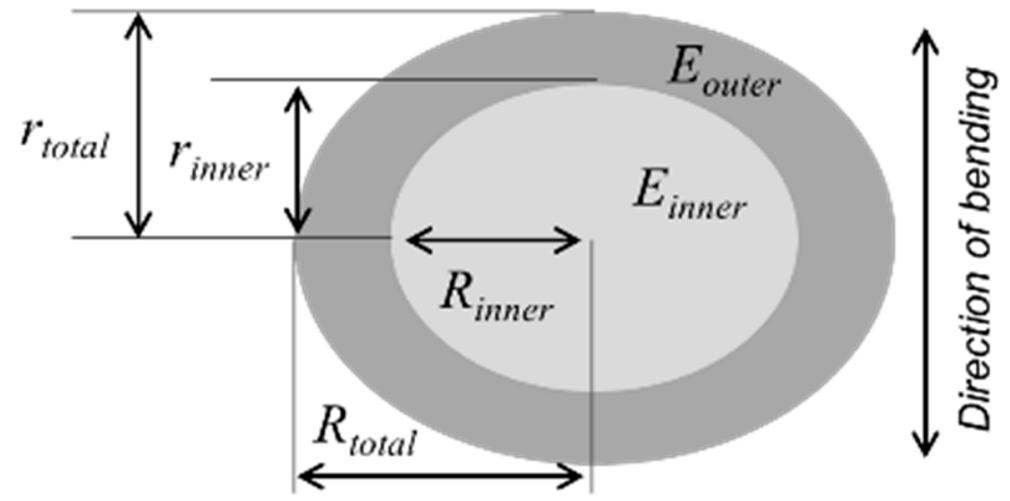

Figure 3. Geometry of double-layered model. $E_{\text {outer }}=$ the bending elasticity of the outer layer, $E_{\text {inner }}=$ the bending elasticity of the inner layer (surface-removed hair), $R_{\text {total }}=$ the hair radius in the major axis of the whole hair, $R_{\text {inner }}=$ the hair radius in the major axis of surface-removed hair, $r_{\text {total }}=$ the hair radius in the minor axis of the whole hair, $r_{\text {inner }}=$ the hair radius in the minor axis of the surface-removed hair.

\subsection{Atomic Force Microscopy (AFM)}

In order to investigate the elasticity of each structure, hair cross-sections were measured using atomic force microscopy (AFM) (MFP-3D-SA-J; Oxford Instruments, Abingdon-on-Thames, Oxfordshire, U.K.). Hair samples embedded in light-curable resins (ARONIX LCR D-800; TOAGOSEI Co., Ltd., Minato, Tokyo, Japan) were sliced with a microtome, as outlined previously. The elasticity of each structure was measured by pushing a cantilever (NCHV; Bruker, Billerica, Massachusetts, USA) into the samples using AFM. The force curve was analyzed according to the Hertz model [23].

\subsection{Differential Scanning Calorimetry (DSC)}

In order to investigate the interaction between organic acid and hair, differential scanning calorimetry (DSC) (DSC7000X; Hitachi High-Tech Science Corporation, Minato, Tokyo, Japan) measurement was performed. Hair samples were stored under $20^{\circ} \mathrm{C}, 65 \% \mathrm{RH}$ for 4 days. An empty pan was used as a reference. Samples were cooled to $-70{ }^{\circ} \mathrm{C}$, followed by a temperature increase to $250^{\circ} \mathrm{C}$ at $10^{\circ} \mathrm{C} / \mathrm{min}$.

\section{Results}

\subsection{Distribution of Cortical Cells}

\subsubsection{TEM Observation of Hair Cross-Sections}

Typical Japanese flexible/elastic and inflexible/inelastic hairs were selected by the sensory tests as described in Table 1 above and the microstructures of those hairs were observed using a TEM.

There were no significant differences in the cuticle or medulla. On the other hand, there were some differences in the cortices. There are two types of cortices in human hair. One is called the "ortho-like cortex", where the intermediate filament (IF) alignment is spiral. It shows relatively small and dispersed macrofibrils in the hair cross-section. The other is the "para-like cortex" where the IF alignment is parallel. It shows relatively large and fused macrofibrils [14-17]. The TEM image of the flexible/elastic hair (sensory score 3 ) is shown in Figure 4 . The flexible/elastic hair shows the characteristic double-layered distribution of the two types of cortical cells (ortho-like and para-like cells). The ortho-like cells (Figure 4a) tend to be located near the hair surface, while the para-like cells 
(Figure $4 \mathrm{~b}$ ) tend to be located near the center of the hair fiber. The thickness of the ortho-like cell layer and cuticle layer are nearly $3 \mu \mathrm{m}$ and $2 \mu \mathrm{m}$, respectively.
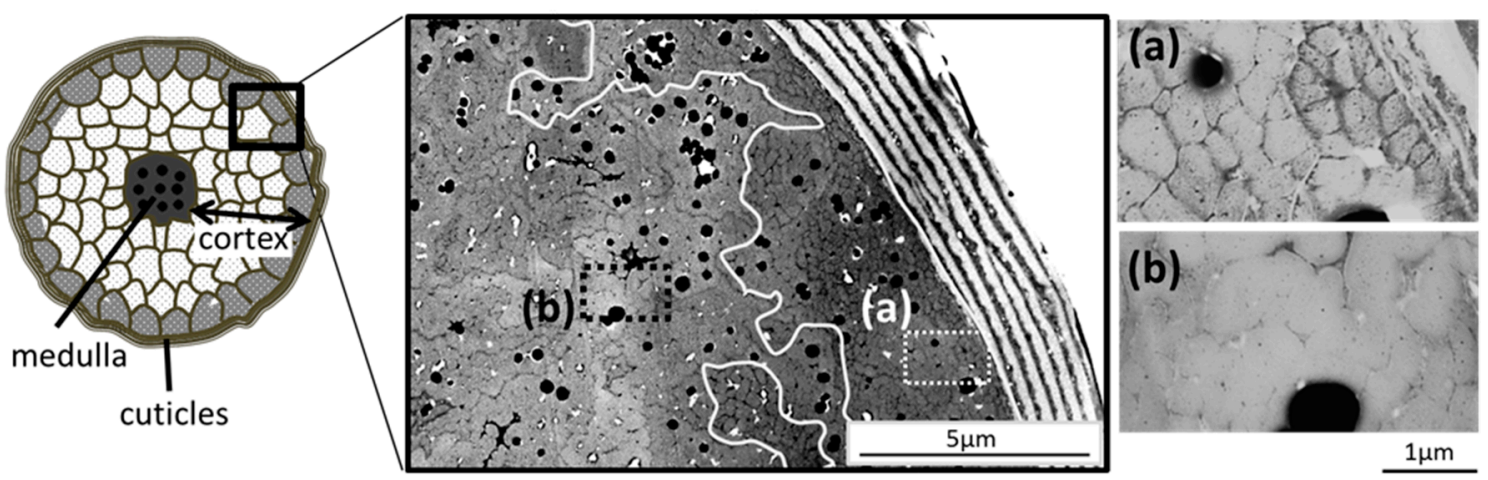

Figure 4. The distribution of two types of cortices in SHINAYAKA (flexible/elastic) hair. (a) Ortho-like cortex, (b) para-like cortex [18].

\subsubsection{FLM Observation of Hair Cross-Sections}

The distributions of the ortho- and para- like cortices in flexible/elastic and inflexible/inelastic hair were confirmed with a differential staining method. One hundred hair samples from each individual (in Table 1) were stained with two types of fluorescent dyes and observed using FLM. Shown in Figure 5 is an image that clearly represents this feature. The flexible/elastic hairs showed the ortho-like cortex distributed near the hair surface (outer layer) and the para-like cortex distributed near the center of the hair fiber (inner layer), as shown in Figure 5a. On the other hand, the inflexible/inelastic hairs show a dispersed pattern of the two types of cortical cells, as shown in Figure 5b, indicating a structural difference between the flexible/elastic hairs and the inflexible/inelastic hairs.

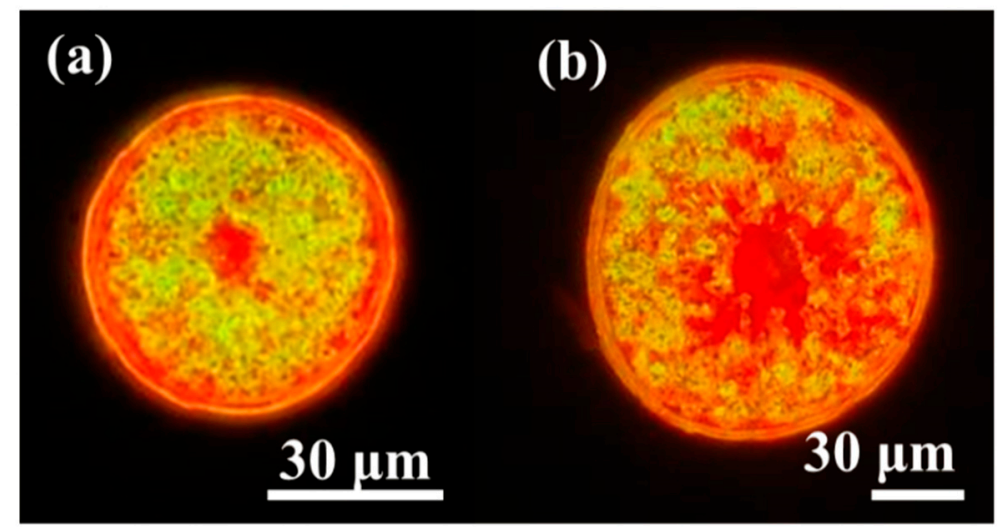

Figure 5. Distribution of two types of cortical cells: (a) flexible/elastic hair (Table 1; No. 1) [18], (b) inflexible/inelastic hair (Table 1; No. 10). The para-like cortical cells look green and the ortho-like cortical cells look red in these pictures.

\subsection{Distribution of Hair Mechanical Properties}

Next, the mechanical differences between flexible/elastic and inflexible/inelastic hair were investigated by various means.

\subsubsection{Bending Elasticity of Outer and Inner Layers}

The results of the bending elasticity of the outer and inner layers obtained with typical flexible/elastic hair (Table 1: No. 1/Sensory Score = 1.6) and inflexible/inelastic hair (Table 1: No. 10/ Sensory Score $=9.3$ ) are shown in Figure 6. These measured values were $E_{\text {outer }}=3.4 \mathrm{GPa}, \mathrm{E}_{\mathrm{inner}}=5.6 \mathrm{GPa}$ and 
$\mathrm{E}_{\text {outer }}=5.1 \mathrm{GPa}, \mathrm{E}_{\text {inner }}=5.3 \mathrm{GPa}$ of each. In this case, the ratios of bending elasticity $\left(\mathrm{E}_{\text {outer }} / \mathrm{E}_{\text {innr }}\right)$ were 0.60 and 0.96 , respectively.

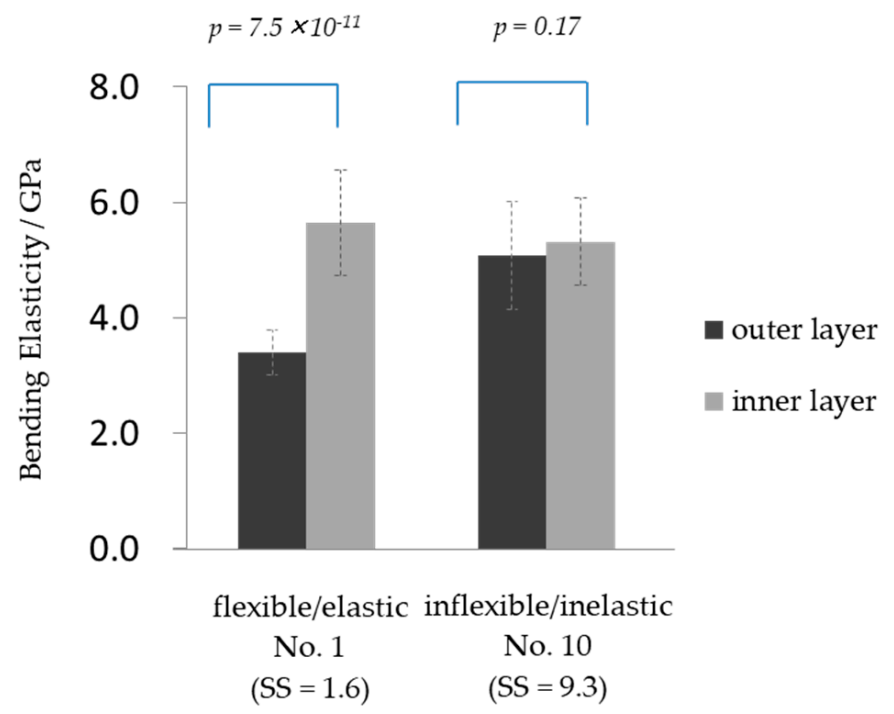

Figure 6. Bending elasticity of the outer and inner layers of flexible/elastic (Table 1; No. 1) and inflexible/inelastic (Table 1 ; No. 10) hairs. Measurements at $20^{\circ} \mathrm{C}$ and $65 \%$ relative humidity (RH), $n=20, \mathrm{SS}=$ sensory score.

The bending elasticity in the outer layer is lower than that in the inner layer and this difference in the flexible/elastic hair is bigger than that in the inflexible/inelastic hair.

\subsubsection{Influence by Hair Damage}

Damaged hair is usually classified as inflexible/inelastic because of its inflexible/inelastic behavior [9-11]. Changes in the mechanical properties between untreated hair and model damaged hair, in the way described above, as well as the root and tip of heavily damaged hair, were compared. The results are shown in Figure 7a,b. When untreated hair (No. 1; described in Table 1) was model damaged as described above, the bending elasticity of the outer layer increased from 3.4 GPa to 4.2 GPa. The ratios of bending elasticity were changed from 0.60 to 0.74 , which is significant. When comparing changes in the mechanical properties between the root and tip of heavily damaged hair, the outer layer of the tip was harder than the root (tip: $4.8 \mathrm{GPa}$, root: $4.2 \mathrm{GPa}$ ). The ratios of bending elasticity were 0.89 (tip) and 0.80 (root). The ratio of the tip was bigger than that of the root. These results suggest that the mechanical property is stiffened by the damage, especially in the outer layer.

(a)

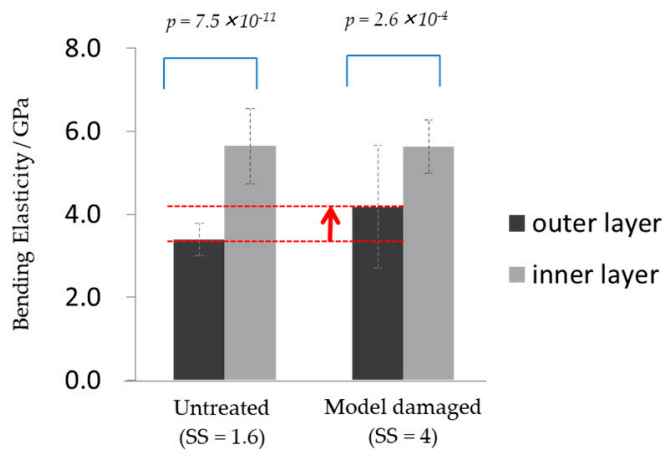

(b)

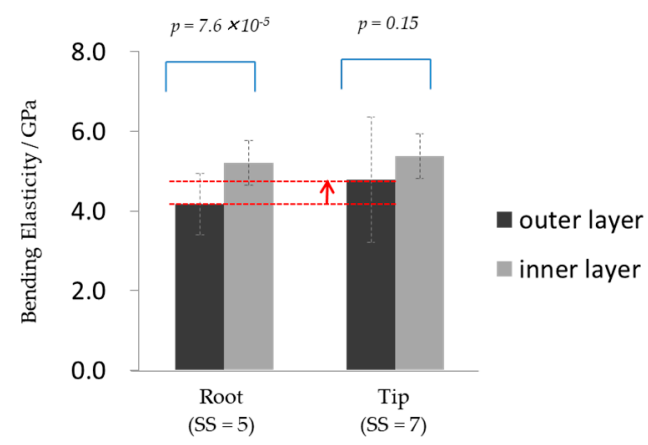

Figure 7. Bending elasticity of the outer and inner layers of healthy hair and damaged hair: (a) flexible/elastic hair (Table 1; No. 1) and model damaged hair (Table 1; No.1). (b) Root and tip of heavily damaged hair. Measurements at $20^{\circ} \mathrm{C}$ and $65 \%$ relative humidity $(\mathrm{RH}), \mathrm{n}=20, \mathrm{SS}=$ sensory score. 


\subsection{Modification of the Mechanical Properties}

The above results suggest that the concentric double-layered distribution of the mechanical properties (softer outer layer and stiffer inner layer) is important for flexible/elastic hair. These results also suggest that the softening of the outer layer is possibly effective for the improvement of hair property from inflexible/inelastic to flexible/elastic. We have studied how to modify the mechanical properties of the hair fiber by investigating the effect of organic acids. Some organic acids have strong interactions with keratinous proteins and can modify mechanical properties [3-8]. Several organic acids, i.e. maleic acid, aspartic acid, or succinic acid, which have hair softening abilities, were tested to investigate whether these acids could selectively soften the outer layer. Succinic acid (SA) was found to be the most effective of these materials.

\subsubsection{Treatment with Succinic Acid}

The colored hair (shown in Section 2.1.2) with model damage treatment (ratio of bending elasticity $=0.99$; sensory score $=7.0$ ) were used. The hair was treated with the solution containing SA by the method described in Section 2.2.3. With this treatment, only the outer layer was softened from 5.4 GPa to 3.7 GPa, and the mechanical difference between the outer layer and the inner layer became clear. At this time, the sensory score changed from 7 to 3. Furthermore, the same tendency was confirmed by the method shown in Sections 2-5 (Figure 8a,b) [18].

(a)

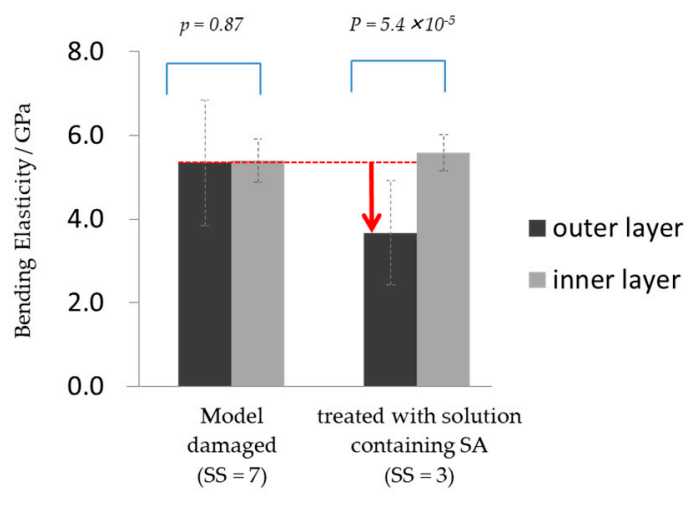

(b)

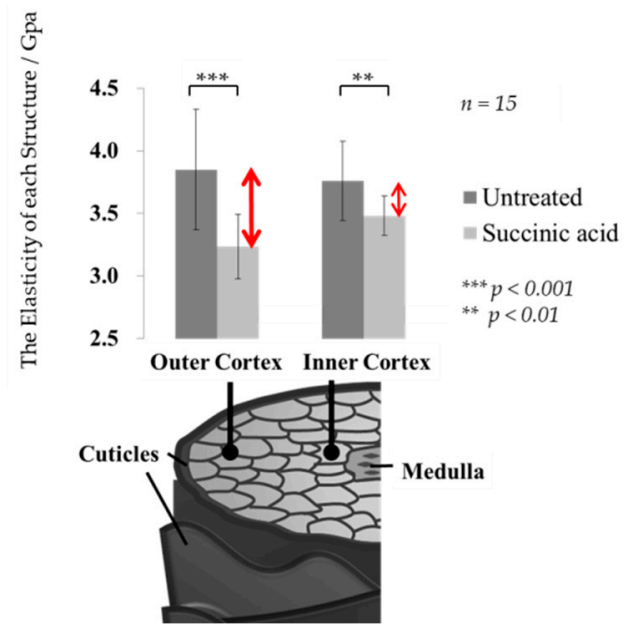

Figure 8. The difference in mechanical properties of model damaged hair and hair treated with a solution containing succinic acid (SA) [18]. (a) Bending elasticity, Measurements at $20{ }^{\circ} \mathrm{C}$ and $65 \%$ relative humidity $(\mathrm{RH}), \mathrm{n}=20, \mathrm{SS}=$ sensory score $(\mathbf{b})$ elasticity of each structure measured using atomic force microscopy (AFM).

\subsubsection{Treatment with the Three-Step Treatment System}

The colored hair with model damage treatment (same as above) and untreated inflexible/inelastic hair (No. 10 shown in Table 1, sensory score = 9.3) were treated with the three-step system using SA. As a result, only the outer layer was softened from 5.4 GPa to 3.7 GPa (above) and from 5.1 GPa to 3.9 GPa (Table 1; No. 10). The difference between the outer layer and the inner layer became more clearly defined (Figure 9), even under realistic usage in in-bath conditions. 
(a)

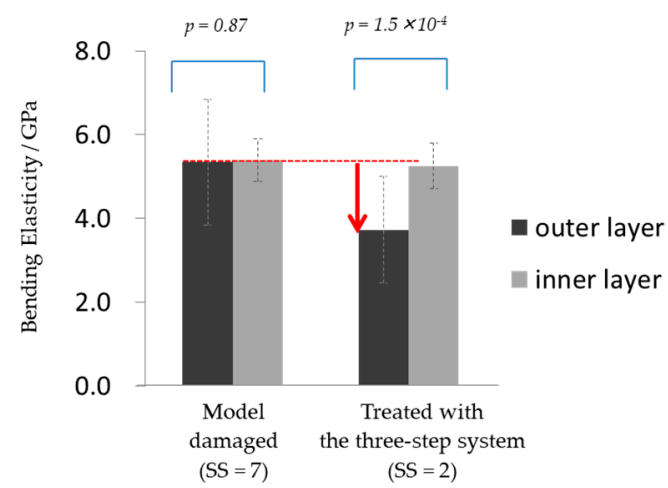

(b)

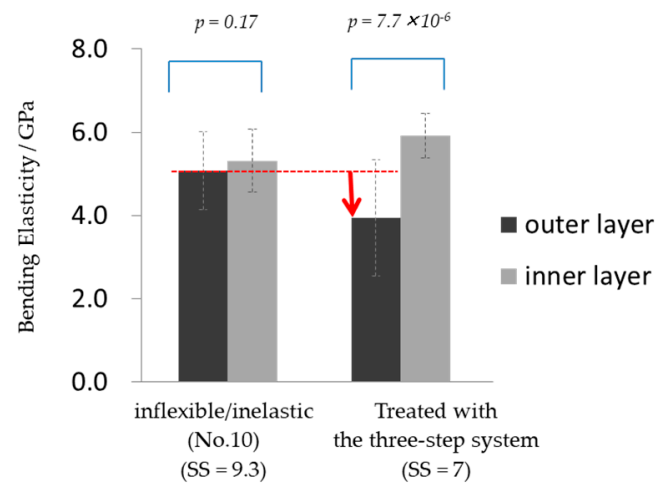

Figure 9. Change in mechanical properties when treated with three-step system using SA. (a) Model damaged hair (b) inflexible/inelastic chemically untreated hair. Measurements at $20^{\circ} \mathrm{C}$ and $65 \%$ relative humidity $(\mathrm{RH}), \mathrm{n}=20, \mathrm{SS}=$ sensory score.

\subsubsection{Application to Caucasian hair}

Similar results were obtained on Caucasian hair. The outer layer became harder after damage (the elasticity of the outer layer increased from 2.9 GPa to $4.8 \mathrm{GPa}$ ), and the outer layer was softened with treatment by the three-step system using SA (the elasticity of the outer layer decreased from $4.8 \mathrm{GPa}$ to $2.8 \mathrm{GPa}$ ) (Figure 10).

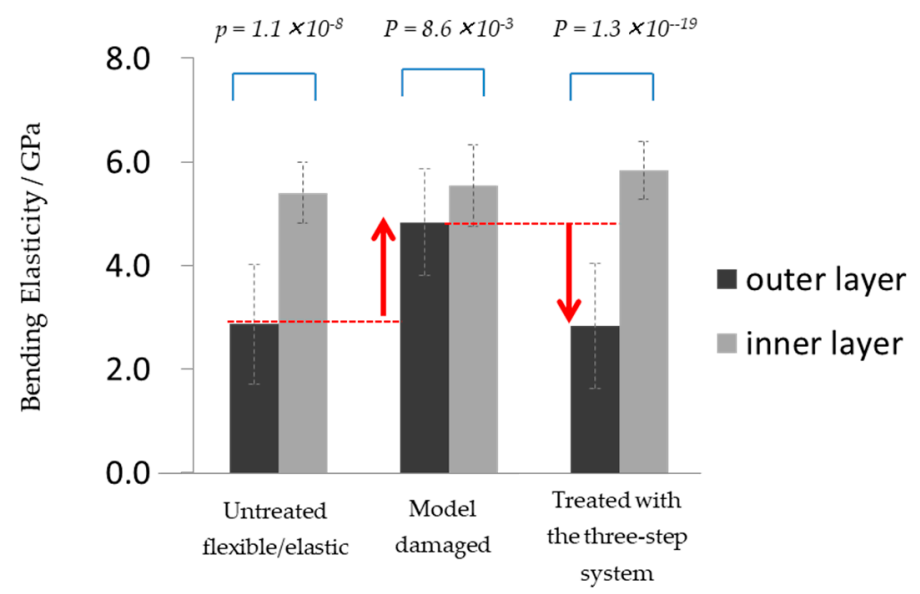

Figure 10. Bending elasticity of the outer and inner layer of Caucasian hair. Measurements at $20{ }^{\circ} \mathrm{C}$ and $65 \%$ relative humidity $(\mathrm{RH}), \mathrm{n}=20$; $\mathrm{SS}=$ sensory score.

\section{Discussion}

\subsection{Characteristic Structural Pattern}

Microscopic observations revealed a structural difference between flexible/elastic hair and inflexible/inelastic hair. For flexible/elastic hair, ortho-like cells tend to concentrate in the outer layers of the hair fiber (near the hair surface), while para-like cells concentrate in the inner layers (near the center). These results indicated that flexible/elastic hair shows a concentric double-layered distribution of cortical cells, as observed by TEM and FLM. In contrast, inflexible/inelastic hair demonstrated a dispersed distribution of the two cortex types.

\subsection{Characteristics of Bending Elasticities}

We considered that flexible/elastic hair not only had structural differences but also had mechanical differences when compared to inflexible/inelastic hair. Generally, a material's structure is closely related to a material's property. The mechanical property of hair should be important for hair motion. 
If we could understand the relationship between mechanical properties and beautiful hair motion, it would be helpful to develop new technologies to control hair motion. So, we investigated the mechanical difference between flexible/elastic and inflexible/inelastic hair. The elasticity of ortho-like cortical cells is relatively lower than that of para-like cortical cells $[24,25]$. Therefore, it is expected that for flexible/elastic hair, the outer layer of the hair fiber is softer than the inner layer. In general, it is understood that the cuticle is the hardest tissue in the hair. Most outer layers of each cuticle cell (called the A-layer) are the hardest tissue in the hair. On the other hand, most inner layers of each cuticle (called the endocuticle) are softer than the cortex. When bending the hair, the softer tissue like the endocuticle can be preferentially deformed, as published previously [19]. Therefore, the contribution of the cuticle to bending elasticity is small.

The bending elasticity was measured by deformation of a straight hair strand into a curled shape with a $25 \mathrm{~mm}$ curl radius for $10 \mathrm{~s}$, creating a deformation velocity of 0.004 per millimeter second. This velocity corresponds to the change in curvature from straight to $220 \mathrm{~mm}$ curl radius in one second. The tip end of the $300 \mathrm{~mm}$ long hair strand moved approximately $170 \mathrm{~mm}$ away within one second. Therefore, the deformation velocity of this system is comparable to the velocity of actual hair swinging while walking and/or turning around (Figure 11).

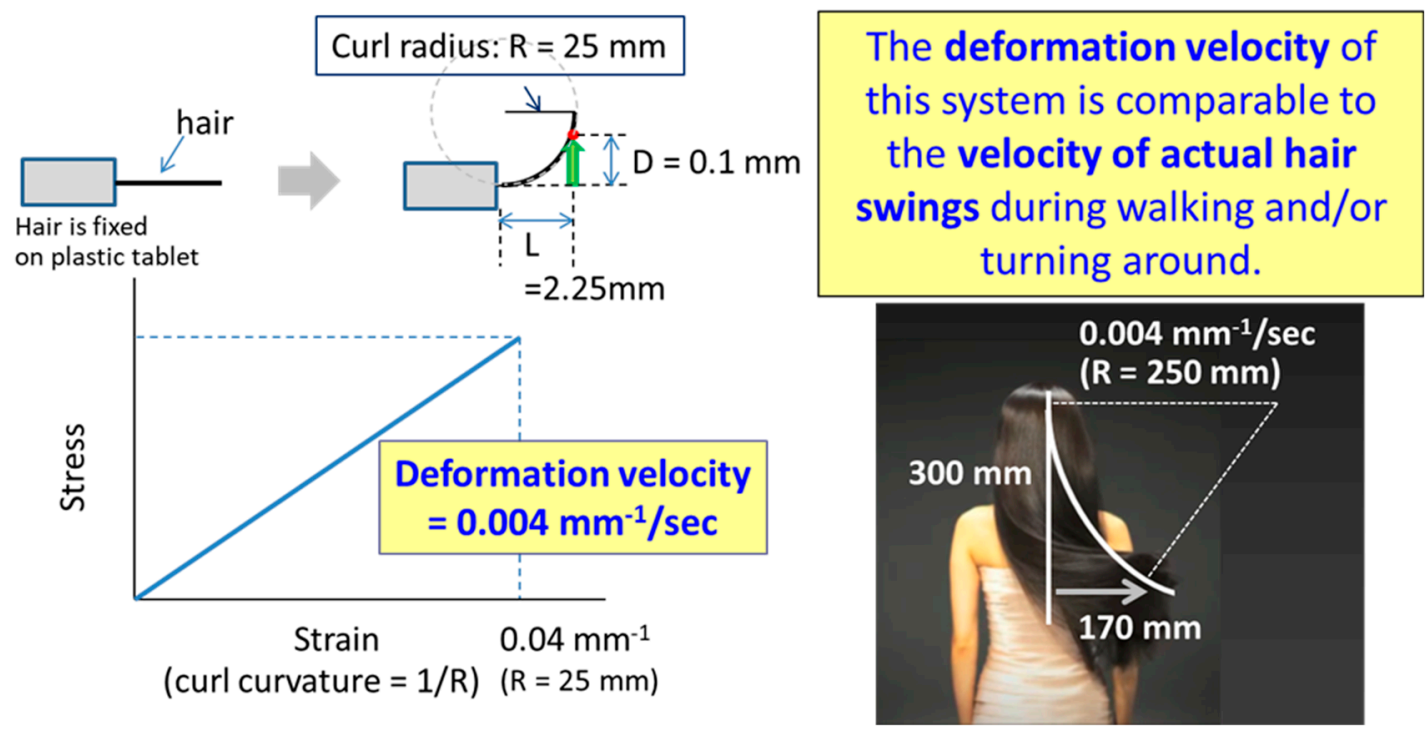

Figure 11. The deformation velocity at the measurement point and the deformation of the actual hair swing.

The results suggest that the bending elasticity in the outer layer is lower than that in the inner layer and this difference between the outer and inner layers in flexible/elastic hair is bigger than in inflexible/inelastic hair. We confirmed that the difference is smaller in damaged hair. In other words, damaged hair did not show the characteristics of the concentric double-layer.

Plotting the ratio of bending elasticity versus sensory score of all samples shown in this report showed a relationship between them. A lower ratio of bending elasticity correlated with a lower sensory score (Figure 12). A lower sensory score indicates a better hair feel and a lower ratio of bending elasticity, indicating a strong separation between the inner and outer layers. The above results suggest that the concentric double-layered distribution of the mechanical properties (softer outer layer and stiffer inner layer) is important for flexible/elastic hair. These results also suggest that softening of the outer layer is possibly effective for the improvement of these hair properties from inflexible/inelastic to flexible/elastic. 


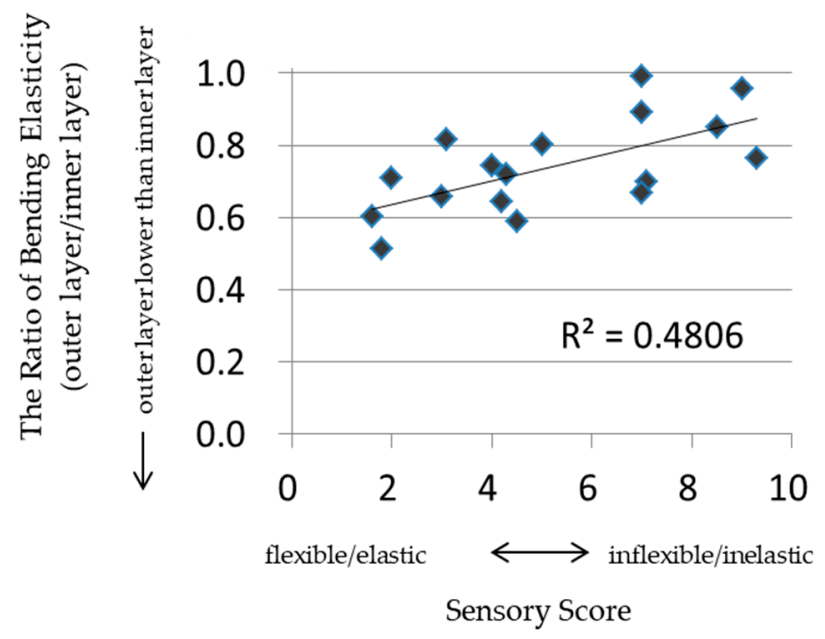

Figure 12. Relationship between the ratio of bending elasticity (outer layer/inner layer) and the sensory score. $\mathrm{R}^{2}=$ coefficient of determination derived from regression line by least squares method.

\subsection{Modification of the Mechanical Properties}

\subsubsection{Effect of Succinic Acid}

We have investigated the effect of organic acids on the mechanical properties of hair fibers. Succinic acid selectively softened the outer layer. We predicted that SA only penetrated the outer layer. On the other hand, malic acid has been found to penetrate deeper into the hair's inner layer [6]. It was hypothesized that succinic acid interacts more strongly with hair proteins and thus likely penetrates less. The interaction between the organic acid and hair proteins was analyzed using DSC. The glass transition temperature $(\mathrm{Tg})$ is shown by the derivative differential scanning calorimetry (DDSC) spectrum at the time of temperature rise (Figure 13). DDSC showed the differential of the DSC spectrum and it could indicate the inflection point more clearly. Both malic acid-treated hair and succinic acid-treated hair decreased in Tg compared to untreated hair. In this case, the Tg of succinic acid treatment decreased more. Assuming that the larger the Tg decrease, the more strongly it interacts with hair proteins, it can be said that succinic acid interacts more strongly with hair proteins compared to malic acid. It follows that succinic acid tends to stay in the surface layer of the hair. In addition, it is thought that succinic acid efficiently softens the proteins, as the decrease in $\mathrm{Tg}$ indicates that water and organic acid plasticized the protein [26-28].

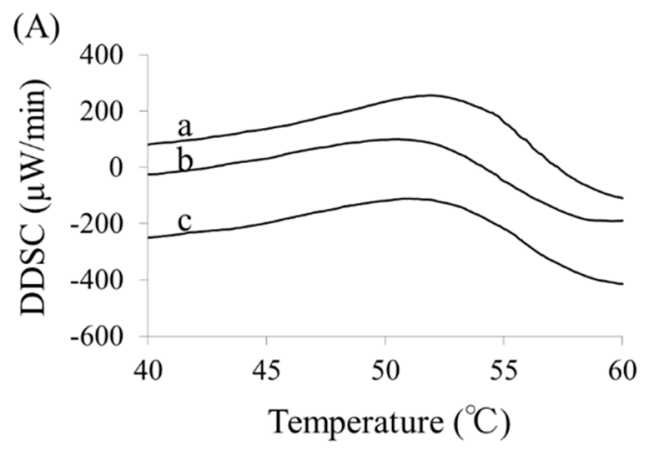

(B)

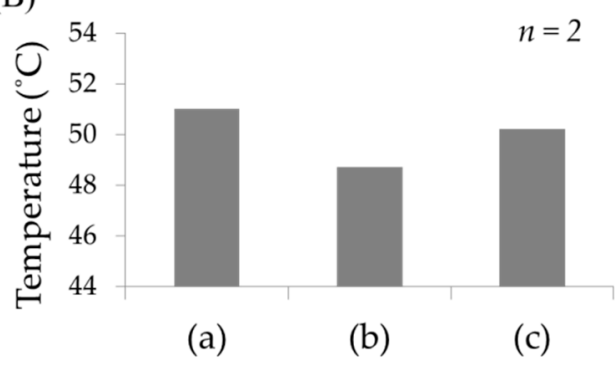

Figure 13. (A) DDSC curves and (B) glass transition temperature of each hair. (a) Without treatment, (b) treated with succinic acid, (c) treated with malic acid [18]. DDSC = derivative differential scanning calorimetry 


\subsubsection{The Three-Step Treatment System}

We tried to add succinic acid (SA) to shampoo and/or conditioner. When SA is added to shampoo, no sufficient effect was obtained, because shampoo is usually rinsed off immediately. When SA is added to a conditioner, no sufficient effect was obtained, because anionic SA is possibly trapped by cationic materials. SA has two pKa values, at 4.2 and at 5.6. The pKa means acid constant expressed in common logarithm. The dissociation state differs depending on $\mathrm{pH}$. Therefore, penetration and solubility can be controlled by $\mathrm{pH}$.

A three-step treatment system was developed. It is composed of shampoo, the first conditioner with SA but without cationic materials, and the second conditioner with cationic materials. We considered that in the second step, SA can easily penetrate into the hair due to its higher solubility at $\mathrm{pH} 6$ without inhibition by cationic materials. In the third step, SA can be fixed into the hair fiber by the cationic materials at $\mathrm{pH}$ 3.4. Based on this system, we have succeeded in controlling the mechanical properties even under realistic usage in in-bath conditions, even when only used one time. The effect of the three-step system was confirmed with Japanese consumers. Perceived changes in hair properties (softness, bounce, flexible and elastic) after use of the three-step treatment system are shown in Figure 14.

Panelists : Japanese women, Age 30-44, $n=56$

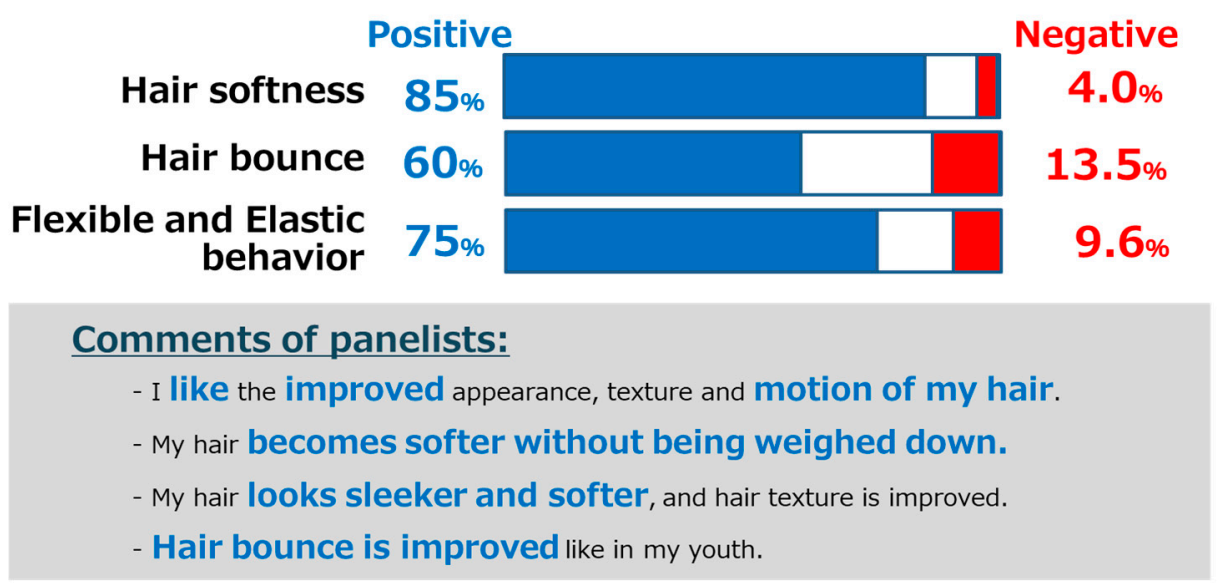

Figure 14. Effect of the three step system confirmed with Japanese consumers. Perceived changes in hair properties when the test formula is used.

\section{Conclusions}

Microscopic observation revealed the concentric double-layered distribution of the two types of cortical cells in flexible/elastic (SHINAYAKA) hair. The ortho-like cortex tends to distribute in the outer layer of the hair (near the hair surface), while the para-like cortex tends to distribute in the inner layer. Based on this observation, it was found that the concentric double-layered distribution of mechanical properties (softer outer layer and stiffer inner layer) is important for the flexible/elastic behavior of the hair. The characteristic mechanical properties were changed due to hair damage and our developing treatment containing SA. It was demonstrated that the mechanical property change occurred not only for Japanese but also Caucasian hair.

Author Contributions: Conceptualization, M.E., T.M., S.N. and T.O.; methodology, M.E., N.T., T.M. and S.N.; investigation, M.E., N.T. and Y.N.; writing, M.E.; supervision, T.O.

Funding: This research received no external funding.

Acknowledgments: We would like to thank E. Terada and K. Koike of KAO Co., Japan, for the useful discussion. We would also like to thank S. Breaksear of KAO Germany GmbH and E. Pyrozoku of KAO Co., Japan, for the useful discussion and his kind English editing for this publication. Special thanks to R. Furukawa for gathering much of the data.

Conflicts of Interest: The authors declare no conflict of interest. 


\section{References}

1. Nagase, S.; Satoh, N.; Nakamura, K. Influence of internal structure of hair fiber on hair appearance. 2. Consideration of the visual perception mechanism of hair appearance. J. Cosmet. Sci. 2002, 53, 387-402. [PubMed]

2. Tanamachi, H.; Inoue, S.; Tanji, N.; Tsujimura, H.; Oguri, M.; Ishita, M.; Tokunaga, S.; Sazanami, F. Deposition of 18-MEA onto alkaline-color-treated weathered hair to form a persistent hydrophobicity. J. Cosmet. Sci. 2009, 60, 31-44. [CrossRef] [PubMed]

3. Okamoto, M.; Yakawa, R.; Mamada, A.; Inoue, S.; Nagase, S.; Shibuichi, S.; Kariya, E.; Satoh, N. Influence of internal structures of hair fiber on hair appearance. 3. Generation of light-scattering factors in hair cuticles and the influence on hair shine. J. Cosmet. Sci. 2003, 54, 353-366. [PubMed]

4. Breakspear, S.; Fukuhara, M.; Itou, T.; Hirano, Y.; Nojiri, M.; Kiyomine, A.; Inoue, S. Alignment control and softness creation in hair with glycylglycine. J. Cosmet. Sci. 2014, 64, 19-33.

5. Nagase, S.; Shibuichi, S.; Ando, K.; Kariya, E.; Okamoto, M.; Yakawa, R.; Mamada, A.; Satoh, N. Light-Scattering Control at Medulla Enhances Human Hair Shine. Internal Structures of Hair Fiber and Its Shine (1). In Proceedings of the 21th IFSCC Congress, Berlin, Germany, 11-14 September 2000.

6. Okamoto, M.; Mamada, A.; Yakawa, R.; Inoue, S.; Nagase, S.; Shibuichi, S.; Satoh, N. Hole Generation Mechanisms in Hair Medulla and Its Repairing Technique. Internal Structures of Hair Fiber and its Shine (3). In Proceedings of the 21th IFSCC Congress, Berlin, Germany, 11-14 September 2000.

7. Nojiri, M.; Itou, T.; Asami, M.; Ueyama, K.; Nakamura, K. A novel technolory for improving hair setting ability and its mechanism. J. Cosmet. Sci. 2004, 55, 151-153.

8. Itou, T.; Nojiri, M.; Ootsuka, Y.; Nakamura, K. Study of interaction between hair protein and organic acid that improves hair-set durability by near-infrared spectroscopy. J. Cosmet. Sci. 2006, 57, 139-151.

9. Galliano, A.; Lheur, M.; Santoprete, R. Analysing the movement of a hair swatch using vdeo and image analysis: Apromibing technique for exploring the dynamic properties of hair. J. Cosmet. Sci. 2015, 37, 56-62. [CrossRef] [PubMed]

10. Hindley, M.C.; Yap, M.; Ugail, H. Measurement of Hair Dynamics via video image Analysis. In Proceedings of the 27th IFSCC Congress, Seoul, Korea, 23-25 October 2017; p. 167.

11. Wiesche, E.S.; Focht, N.; Wortmann, F.J. The Swinging Behaviour of Human Hair-A Novel Method to Quantify Hair Collective Movements. In Proceedings of the 23rd IFSCC Conference, Zurich, Switzerland, 21-23 September 2015; p. 123.

12. Obataya, E.; Kitin, P.; Yamauchi, H. Bending characteristics of bamboo (Phyllostachys pubescens) with respect to its fiber-foam composite structure. Wood Sci. Technol. 2007, 41, 385-400. [CrossRef]

13. Balfour, H. Structure and affinities of the composite bow. J. Anthr. Inst. Great Br. Irel. 1890, 19, $220-250$. [CrossRef]

14. Bryson, W.G.; Harland, D.P.; Caldwell, J.P.; Vernon, J.A.; Walls, R.J.; Woods, J.L.; Nagase, S.; Itou, T.; Koike, K. Cortical cell types and intermediate filament arrangements correlate with fiber curvature in Japanese human hair. J. Struct. Biol. 2009, 166, 46-58. [CrossRef]

15. Kajiura, Y.; Watanabe, S.; Itou, T.; Nakamura, K.; Iida, A.; Inoue, K.; Yagi, N.; Shinohara, Y.; Amemiya, Y. Structural analysis of human hair single fibers by scanning microbeam SAXS. J. Struct. Biol. 2006, 155, 438-444. [CrossRef] [PubMed]

16. Nagase, S.; Tsuchiya, M.; Matsui, T.; Shibuichi, S.; Tsujimura, H.; Masukawa, Y.; Satoh, N.; Itou, T.; Koike, K.; Tsujii, K. Characterization of curved hair of Japanese women with reference to internal structures and amino acid composition. J. Cosmet. Sci. 2008, 59, 317-332. [PubMed]

17. Nagase, S.; Kajiura, Y.; Mamada, A.; Abe, H.; Shibuichi, S.; Satoh, N.; Itou, T.; Shinohara, Y.; Amemiya, Y. Changes in structure and geometric properties of human hair by aging. J. Cosmet. Sci. 2009, 60, 637-648. [CrossRef] [PubMed]

18. Nishita, Y.; Ezure, M.; Tanji, N.; Mizooku, T.; Nagase, S.; Osumi, T. Physical Properties of Shinayaka Hair and Fine Technology to Control Internal Hair Properties. J. Soc. Cosmet. Jpn. 2017, 51, 126-133. [CrossRef]

19. Breakspear, S.; Mamada, A.; Itou, T.; Noecker, B. Contribution of the cuticle to the stiffness hair: Significant or minor? IFSCC Mag. 2015, 1, 25-34.

20. Swift, J.A. Some simple theoretical considerations on the bending stiffness of human hair. Int. J. Cos. Sci. 1995, 17, 245-253. [CrossRef] [PubMed] 
21. Bucciarelli, L.L. Engineering Mechanics for Structures; Dover Publications, INC: Mineola, NY, USA, 2009; pp. 263-271.

22. Sogabe, A.; Yasuda, M.; Noda, A. Physical properties of human hair 1. Evaluation of bending stress by measuring the major and the minor axis of human hair. J. Soc. Cosmet. Chem. Jpn. 2002, 36, 207-215. [CrossRef]

23. Nakajima, K.; Fujinami, S.; Nukaga, H.; Watabe, H.; Kitano, H.; Ono, N.; Endoh, K.; Kaneko, M.; Nishi, T. Nanorheology mapping by atomic force microsdopy. Kobunshi Ronbunshu 2005, 63, 476-487. [CrossRef]

24. Caldwell, J.P.; Bryson, W.G. Elastic modulus mapping of the wool fibre cellular structure by atomic force microscopy. In Proceedings of the 11th International Wool Research Conference, Leeds, UK, 4-9 September 2005; p. 89FWS.

25. Ezawa, Y.; Nagase, S.; Mamada, A.; Inoue, S.; Koike, K.; Itou, T. Stiffness of human hair correlates with the fractions of cortical cell types. Cosmetics 2019, 6, 24. [CrossRef]

26. Wortmann, F.J.; Stapels, M.; Elliott, R.; Chandra, L. The effect of water on the glass transition of human hair. Biopolymers 2006, 81, 371-375. [CrossRef]

27. Gao, T. Evaluation of hair humidity resistance/moisturization from hair elasticity. J. Cosmet. Sci. 2007, 58, 393-404. [PubMed]

28. Scott, G.V.; Robbins, C.R. Stiffness of human hair fibers. J. Soc. Cosmet. Chem. 1978, 29, 469-485.

(C) 2019 by the authors. Licensee MDPI, Basel, Switzerland. This article is an open access article distributed under the terms and conditions of the Creative Commons Attribution (CC BY) license (http://creativecommons.org/licenses/by/4.0/). 